\title{
India's Trade Performance in Poultry Products
}

\author{
Priyanka Agarwal*, Rakesh Singh, Harshita Tewari and Rekha Rani
}

\author{
Department of Agricultural Economics, Institute of Agricultural Sciences, BHU, \\ Varanasi-221005, U.P., India \\ *Corresponding author
}

\begin{abstract}
A B S T R A C T
Poultry is one of the fastest growing segments of the agricultural sector. Production of eggs and broilers has been rising at a rate of 8 to 10 percent per annum. In term of export from India, Poultry Products recorded 18\% growth during the financial year 2015-16 (APEDA). Therefore the study was conducted with an aim to capture the dynamics of the poultry sector at international level by studying the trade composition and performance of poultry sector, diversity in export import of poultry products and comparative advantage of India's poultry sector export. The study is based on time series data pertaining to the period 1996-2013. The data on value of exports/imports for India and world were collected from FAOSTAT database.

The data was analysed using simple descriptive statistical tools. Simpson diversification index was used to measure the export import diversity and export performance ratio were calculated to find out the comparative advantage. The results revealed that Eggs hen in shell comprises the highest per cent of the poultry products exported from India followed by the Egg dried. However, there was no export of Offal during study period. Both Live Turkey and turkey meat comprises almost negligible share in export value of total poultry products from India. No live poultry were imported to India during triennium ending (T.E.) 2001. Egg hen in shell comprises highest share in import value of poultry sector while no import of eggs other birds in shell during 1996-2013. Among offals, only offals liver ducks were imported to India during 200513.Share of import and export value of turkey in total value of live poultry imported and exported for India shows changing status of India from importer of turkey to exporter of turkey. It was found that chicken was the most frequently traded live poultry product. The trend in net export shows that overall India enjoyed as net exporter of total poultry products. The trend in growth rate shows that egg hen in shell and egg liquid shows positive import growth rate for 1996-2013. Maximum export variability was exhibits by live Turkey for period 1996 to 2004 and maximum imports variability was for meat chicken in both sub-period 1996-2004 and 2005-13. It was found that there is relatively more trade diversity in world than India. The value to import diversification index for world was 0.92 in T.E. 2013 showing almost complete import diversification of poultry sector. Maximum diversification in import of poultry products in India was observed during T.E. 2007. India has comparative advantage Eggs dried, Eggs hen in shell. However India lost comparative advantage in egg liquids during 2005 to 2010. It is suggested that India should make efforts for diversification.
\end{abstract}

\section{Keywords}

Poultry, Simpson index, Comparative advantage,

Diversification,

Export Performance Ratio.

Article Info

Accepted: 07 September 2017 Available Online: 10 October 2017

\section{Introduction}

Animal Products plays an important role in the socio- economic life of India. India's exports of Animal Products were Rs. 30,137.08 Crores in 2015-16. Now a day poultry is one of the fastest growing segments of the agricultural sector. Production of eggs and broilers has been rising at a rate of 8 to 10 percent per annum. In term of export from 
India, Poultry Products recorded 18\% growth during the financial year 2015-16 over the same period of last year (APEDA). There is much more scope of new international markets as poultry meat is the fastest growing component of global meat demand, and India, the world's second largest developing country and is experiencing rapid growth in its poultry sector. India has exported 6,59,304.15 MT of Poultry products to the world for the worth of Rs. 768.72 crores during the year 201516(APEDA).

There is need to examine the recent past performance of the poultry sector at international level so that new dimensions can be touched. Therefore the present study was conducted with aim to capture the dynamics of the poultry sector at international level. Following main objectives were framed

To study the composition of trade of poultry sector

To analyse the trade performance of the poultry sector

To measure the diversity in export import of poultry products

To measure the comparative advantage of India's poultry sector export based on observed trade pattern.

\section{Materials and Methods}

The poultry products are exported imported either in form of live animals or as egg, meat, canned meats offals livers etc. In the present paper Eggs dried, Eggs hen in shell, Egg liquid, Eggs other bird in shell, Meat chicken, Meat chicken canned, Meat duck, Meat goose and guinea fowl, Meat turkey, Offals liver chicken, Offals liver duck, Offals liver geese, Chickens, Ducks and Turkeys poultry products were taken to carry out the study, of which Chicken, Ducks and Turkey were under live poultry products.

The study is based on time series data pertaining to the period 1996-2013. The data on value (in terms of 1000 US\$) of exports/imports for India and world were collected from FAOSTAT database.

The results were based on comparison among the triennium 1996-97, 1999-2001, 2002-04, 2005-07, 2008-10 and 2011-13. To study the composition of trade of poultry sector simple descriptive statistical tools were used, trade performance of the poultry sector was analysed net export, compound annual growth rate and coefficient of variation were calculated. The diversity in export and import of poultry sector was analyzed using Simpson diversification index.

Simpson Index $=1-\Sigma \mathrm{A}_{\mathrm{i}}{ }^{2} / \mathrm{A}^{2}$

Where,

$A_{i}=$ Value of export/import of $i^{\text {th }}$ livestock product

$\mathrm{A}=$ Value of export/import of total livestock products.

The value of the index varies from 0 to 1.1 indicates total diversification, whereas a value of 0 indicates perfect concentration of trade towards a particular product in the particular triennium.

To measures the comparative advantage of India's Poultry sector exports, Export Performance Ratio (EPR), as suggested by Balassa (1965) was used. The EPR of the $i^{\text {th }}$ Poultry product $\left(\mathrm{EPR}_{\mathrm{i}}\right)$ can be expressed as:

$\mathrm{EPR}_{\mathrm{i}}=\left(\mathrm{E}_{\mathrm{i}} / \mathrm{CE}\right) /\left(\mathrm{W}_{\mathrm{i}} / \mathrm{WE}\right)$

Where,

$E_{i}=$ Export of $i^{\text {th }}$ Poultry product from India 
$\mathrm{CE}=$ Aggregate export of Poultry products from India

$\mathrm{Wi}=$ Total world export of $\mathrm{i}^{\text {th }}$ Poultry product

$\mathrm{WE}=$ Total world export of all Poultry products

A value of EPR greater than unity implies that India has comparative advantage in the exports of particular Poultry products and vice versa

\section{Results and Discussion}

Table 1 depicts the contribution of poultry export/import to total export/import value of livestock sector in India. The share of export and import values from poultry sector to livestock sector shows almost similar type of trend. Initially, share of export from poultry sector shows increasing trend till triennium ending (T.E.) 2007, then starts to decline and reaches to 31.79 percent in T.E. 2013, showing significant fall in share of export value of poultry. Similarly share of import shows increasing trend till T.E. 2010 then decrease to 1.40 per cent in T.E. 2013. However there is fall in share of import during 2011-13 but it was greater than its share during end 90 's.

\section{Composition and trend of trade of poultry sector}

Table 2 shows the export value of Indian poultry sector during 1996 to 2013 in 1000 US\$. More than 95 per cent of the export value of total poultry sector was contributed by other poultry products while export value of live poultry was considerably low. Other Poultry products showed increasing trend in export however live poultry shows decreasing trend except for the T.E.2001.The share of other poultry products to total export of poultry products was around 99 per cent during 2002 to 2013. During the study period,
Eggs hen in shell comprises the highest percent of the poultry products exported from India followed by the Egg dried. However Eggs other birds in shell were exported only in the T.E. 2013. It was also observed that there was no export of Offal during 1996 to 2013. Meat, goose and guinea fowl were not exported till 2010 and formed negligible amount in the T.E. 2013. Both Live Turkey and turkey meat comprises almost negligible share in export value of total poultry products from India. There are fluctuations in share of export of both canned chicken meats and chicken meat. The share of meat chicken canned shows decreasing trend till T.E. 2007, of which more than 50 percent decline in share was observed from T.E. 1998 to T.E. 2001. Share of chicken meat shows increasing trend except for T.E. 2007. From 2002 onwards the share of chicken meat was relatively higher than the canned chicken meat this may be the outcome of lesser demand of Indian processed chicken meat in international market.

The import value for India poultry sector is compiled in Table 3. The share of other poultry products in total import value of poultry shows first increasing then decreasing trend, its share rises to 100 per cent in T.E. 2001, showing no live poultry were imported to India during T.E. 2001. In T.E. 1998, 89.28 per cent of the total value of poultry import was formed by other poultry product while, the share of live poultry was 10.71 per cent. Share of live poultry was highest in T.E. 2010 which was found to be 83.68 per cent of the total import value of poultry products.

Egg hen in shell comprises highest share in import value of poultry sector. However there was no import of eggs other birds in shell during 1996-2013. It was also observed that no offals were imported to India except of offals liver ducks, which were imported to India during 2005-13. Share of import value of turkey shows a decreasing trend, in T.E. 
1998 only turkey was imported to India while no live turkey were imported during 1999. 2004 and 2008-10.

Figures 1 and 2 reflect the composition of export and import value of live poultry products respectively. Share of import and export value of turkey in total value of live poultry import and export of India shows that among all live poultry product, only turkey was imported to India in T.E.1998 after which its share is almost negligible and export turkey started from India from the triennium 2002-04 and shows an increasing trend, thus it can be concluded that there is change in status of India from importer of turkey to exporter of turkey. Share of Ducks in export values of live poultry shows increasing and then decreasing trend with highest peak in T.E. 2001. In triennium 20011-13 ducks comprises only 1.09 per cent of the total value of live poultry exported from India whereas 83.82 per cent of the total value of live poultry animals that were imported to India were chicken.

The export value composition of live poultry shows that only ducks and chickens were exported during 1996 to 2004 of which initially ducks formed greatest share in total value of live poultry exported from India till T.E. 2004 after which chicken was mostly exported. Share of chicken in import value of live poultry, constantly shows an increasing trend. Thus showing that chicken were the most frequently traded live poultry product.

\section{Trend in net exports}

Table 4 reveals the trend in net export of poultry during the reference period. The negative values of net export reflect the status of net importer.

It was found that India is net exporter of total poultry products however the status varies from product to product, as for Egg dried, Egg hen in shell, Egg liquid, Meat chicken, Meat chicken canned India is net exporter during the study period. For meat duck India is net exporter except triennium 1999-2001 in which there was net import of meat duck. During period 2008-10 India was net importer of meat turkey, offals liver duck, chickens and ducks. For T.E. 2004 and 1998 India was net importer of live turkey only and for T.E. 2004 India is found to be net exporter for all the poultry product.

Table.1 Share of Poultry sector to total livestock traded from India (per cent)

\begin{tabular}{|l|l|l|l|}
\hline S. No. & Years & Export & Import \\
\hline 1. & $1996-98$ & 46.55 & 0.02 \\
\hline 2. & $1999-2001$ & 48.61 & 0.14 \\
\hline 3. & $2002-04$ & 63.30 & 0.90 \\
\hline 4. & $2005-07$ & 69.51 & 2.18 \\
\hline $\mathbf{5 .}$ & $2008-10$ & 36.53 & 2.64 \\
\hline 6. & $2011-13$ & 31.79 & 1.40 \\
\hline
\end{tabular}


Table.2 Export (Value) of live and other poultry products from India (1000 US\$)

\begin{tabular}{|c|c|c|c|c|c|c|c|}
\hline \multirow[b]{2}{*}{ S. No. } & \multirow[b]{2}{*}{ Items $(\mathbf{X})$} & \multicolumn{6}{|c|}{ Average Export Value of Commodity (1000 US\$) } \\
\hline & & 1996-98 & 1999-2001 & 2002-04 & 2005-07 & 2008-10 & 2011-13 \\
\hline 1. & \multicolumn{7}{|c|}{ Other Poultry Products } \\
\hline i) & Eggs, dried & $\begin{array}{c}6959.33 \\
(27.24)\end{array}$ & $\begin{array}{c}3340.67 \\
(13.95)\end{array}$ & $\begin{array}{c}8625 \\
(16.24) \\
\end{array}$ & $\begin{array}{c}31981 \\
(38.29)\end{array}$ & $\begin{array}{c}31567.33 \\
(38.23)\end{array}$ & $\begin{array}{c}37457.33 \\
(40.01)\end{array}$ \\
\hline ii) & Eggs, hen, in shell & $\begin{array}{c}12847 \\
(50.29)\end{array}$ & $\begin{array}{c}12477 \\
(52.11)\end{array}$ & $\begin{array}{l}27045 \\
(50.93)\end{array}$ & $\begin{array}{c}47887.33 \\
(57.33)\end{array}$ & $\begin{array}{c}46705.67 \\
(56.57)\end{array}$ & $\begin{array}{c}41113 \\
(43.92)\end{array}$ \\
\hline iii) & Eggs, liquid & $\begin{array}{c}4076.33 \\
(15.95) \\
\end{array}$ & $\begin{array}{c}6753 \\
(28.20) \\
\end{array}$ & $\begin{array}{l}13188 \\
(24.84) \\
\end{array}$ & $\begin{array}{c}1072 \\
(1.28) \\
\end{array}$ & $\begin{array}{c}900 \\
(1.09)\end{array}$ & $\begin{array}{c}2132.67 \\
(2.28) \\
\end{array}$ \\
\hline iv) & $\begin{array}{l}\text { Eggs, other bird, in } \\
\text { shell }\end{array}$ & 0 & 0 & 0 & 0 & 0 & $\begin{array}{c}1618 \\
(1.72)\end{array}$ \\
\hline v) & Meat, chicken & $\begin{array}{c}10.33 \\
\#\end{array}$ & $\begin{array}{l}47.67 \\
(0.19) \\
\end{array}$ & $\begin{array}{c}2078.67 \\
(3.91) \\
\end{array}$ & $\begin{array}{c}652.67 \\
(0.78) \\
\end{array}$ & $\begin{array}{c}1870.333 \\
(2.65)\end{array}$ & $\begin{array}{c}9203.67 \\
(9.83) \\
\end{array}$ \\
\hline vi) & $\begin{array}{l}\text { Meat, chicken, } \\
\text { canned }\end{array}$ & $\begin{array}{c}632 \\
(2.47)\end{array}$ & $\begin{array}{c}244.33 \\
(1.02)\end{array}$ & $\begin{array}{c}366.33 \\
(0.69)\end{array}$ & $\begin{array}{c}495 \\
(0.59)\end{array}$ & $\begin{array}{c}591 \\
(0.71)\end{array}$ & $\begin{array}{c}611 \\
(0.65)\end{array}$ \\
\hline vii) & Meat, duck & $\begin{array}{l}9 \\
\# \\
\end{array}$ & $\begin{array}{l}1 \\
\# \\
\end{array}$ & $\begin{array}{c}349 \\
(0.66) \\
\end{array}$ & $\begin{array}{c}176.67 \\
(0.21) \\
\end{array}$ & $\begin{array}{c}586 \\
(0.70) \\
\end{array}$ & $\begin{array}{c}764 \\
(0.81) \\
\end{array}$ \\
\hline viii) & $\begin{array}{l}\text { Meat, goose and } \\
\text { guinea fowl }\end{array}$ & 0 & 0 & 0 & 0 & 0 & $\begin{array}{c}0.67 \\
\#\end{array}$ \\
\hline ix) & Meat, turkey & 0 & 0 & $\begin{array}{c}326.33 \\
(0.61) \\
\end{array}$ & $\begin{array}{c}716.33 \\
(0.86) \\
\end{array}$ & 0 & $\begin{array}{c}91.33 \\
\#\end{array}$ \\
\hline $\mathrm{x})$ & Offals, liver chicken & 0 & 0 & 0 & 0 & 0 & 0 \\
\hline $\mathrm{xi})$ & Offals, liver duck & 0 & 0 & 0 & 0 & 0 & 0 \\
\hline xii) & Offals, liver geese & 0 & 0 & 0 & 0 & 0 & 0 \\
\hline & Total & $\begin{array}{c}24533.99 \\
(96.05) \\
\end{array}$ & $\begin{array}{c}22863.67 \\
(95.48) \\
\end{array}$ & $\begin{array}{c}51978.33 \\
(97.88) \\
\end{array}$ & $\begin{array}{r}82981 \\
(99.35) \\
\end{array}$ & $\begin{array}{c}82220.33 \\
(99.59) \\
\end{array}$ & $\begin{array}{c}92991.66 \\
(99.34) \\
\end{array}$ \\
\hline 2 & \multicolumn{7}{|l|}{ Live Poultry } \\
\hline i) & Chickens & $\begin{array}{c}328.67 \\
(1.28)\end{array}$ & $\begin{array}{c}312.33 \\
(1.30)\end{array}$ & $\begin{array}{c}449.67 \\
(0.85)\end{array}$ & $\begin{array}{c}290.67 \\
(0.34)\end{array}$ & $\begin{array}{c}183.67 \\
(0.22)\end{array}$ & $\begin{array}{c}400.67 \\
(0.42)\end{array}$ \\
\hline ii) & Ducks & $\begin{array}{c}681 \\
(2.66) \\
\end{array}$ & $\begin{array}{c}769.67 \\
(3.21) \\
\end{array}$ & $\begin{array}{c}672.67 \\
(1.27) \\
\end{array}$ & $\begin{array}{c}185.67 \\
(0.22) \\
\end{array}$ & $\begin{array}{c}74.67 \\
\# \\
\end{array}$ & $\begin{array}{c}6.67 \\
\# \\
\end{array}$ \\
\hline iii) & Turkeys & 0 & 0 & $\begin{array}{c}1.67 \\
\#\end{array}$ & $\begin{array}{c}66 \\
\#\end{array}$ & $\begin{array}{c}77.67 \\
\#\end{array}$ & $\begin{array}{c}203.67 \\
(0.21)\end{array}$ \\
\hline & Total & $\begin{array}{c}1009.67 \\
(3.95) \\
\end{array}$ & $\begin{array}{r}1082 \\
(4.52) \\
\end{array}$ & $\begin{array}{c}1124 \\
(2.12) \\
\end{array}$ & $\begin{array}{c}542.33 \\
(0.65) \\
\end{array}$ & $\begin{array}{c}336 \\
(0.40) \\
\end{array}$ & $\begin{array}{c}611 \\
(0.65) \\
\end{array}$ \\
\hline & Grand Total & $\begin{array}{c}25543.66 \\
(100)\end{array}$ & $\begin{array}{c}23945.67 \\
(100)\end{array}$ & $\begin{array}{c}53102.33 \\
(100)\end{array}$ & $\begin{array}{c}83523.33 \\
(100)\end{array}$ & $\begin{array}{c}82556.33 \\
(100)\end{array}$ & $\begin{array}{c}93602.66 \\
(100)\end{array}$ \\
\hline
\end{tabular}

Figure in parenthesis indicates percentage of total export value of poultry

\# indicates negligible share 
Table.3 Import (Value) of live poultry and poultry products from India (1000 US\$)

\begin{tabular}{|c|c|c|c|c|c|c|c|}
\hline & & \multicolumn{6}{|c|}{ Average Import Value of Commodity (1000 US\$) } \\
\hline $\begin{array}{l}\text { S. } \\
\text { No. }\end{array}$ & Items & $\begin{array}{l}1996- \\
98\end{array}$ & 1999-01 & 2002-04 & 2005-07 & 2008-10 & 2011-13 \\
\hline 1. & \multicolumn{7}{|c|}{ Other Poultry Products } \\
\hline i) & Eggs, dried & 0 & 0 & $\begin{array}{l}52 \\
(6)\end{array}$ & $\begin{array}{c}60 \\
(2.023)\end{array}$ & 0 & $\begin{array}{c}5.67 \\
(0.15)\end{array}$ \\
\hline ii) & $\begin{array}{l}\text { Eggs, hen, in } \\
\text { shell }\end{array}$ & $\begin{array}{c}13.67 \\
(73.21)\end{array}$ & $\begin{array}{c}63.67 \\
(67.49)\end{array}$ & $\begin{array}{l}614.67 \\
(70.95)\end{array}$ & $\begin{array}{l}691.67 \\
(23.32)\end{array}$ & $\begin{array}{l}435.33 \\
(10.31)\end{array}$ & $\begin{array}{c}1062 \\
(28.76)\end{array}$ \\
\hline iii) & Eggs, liquid & $\begin{array}{c}0.33 \\
(1.79)\end{array}$ & 0 & $\begin{array}{l}34.33 \\
(3.96)\end{array}$ & $\begin{array}{l}16.33 \\
(0.55)\end{array}$ & $\begin{array}{l}59.67 \\
(1.41)\end{array}$ & $\begin{array}{l}5.33 \\
(0.14)\end{array}$ \\
\hline iv) & $\begin{array}{l}\text { Eggs, other } \\
\text { bird, in shell }\end{array}$ & 0 & 0 & 0 & 0 & 0 & 0 \\
\hline v) & Meat, chicken & 0 & 0 & $\begin{array}{c}2.67 \\
(0.31)\end{array}$ & $\begin{array}{c}9.33 \\
(0.31)\end{array}$ & 0 & 0 \\
\hline vi) & $\begin{array}{l}\text { Meat, chicken, } \\
\text { canned }\end{array}$ & $\begin{array}{c}0.67 \\
(3.57) \\
\end{array}$ & $\begin{array}{c}25.67 \\
(27.21) \\
\end{array}$ & $\begin{array}{c}35 \\
(4.04) \\
\end{array}$ & $\begin{array}{l}69.33 \\
(2.34) \\
\end{array}$ & $\begin{array}{l}175.33 \\
(4.15) \\
\end{array}$ & $\begin{array}{c}221.67 \\
(6)\end{array}$ \\
\hline vii) & Meat, duck & $\begin{array}{c}2 \\
(10.71)\end{array}$ & $\begin{array}{c}3.67 \\
(3.89)\end{array}$ & $\begin{array}{c}4.67 \\
(0.54)\end{array}$ & $\begin{array}{l}18.67 \\
(0.62)\end{array}$ & $\begin{array}{l}4.33 \\
(0.10)\end{array}$ & $\begin{array}{l}72.33 \\
(1.96)\end{array}$ \\
\hline viii) & $\begin{array}{l}\text { Meat, goose } \\
\text { and guinea fowl }\end{array}$ & 0 & 0 & 0 & 0 & 0 & 0 \\
\hline ix) & Meat, turkey & 0 & $\begin{array}{c}1.33 \\
(1.41)\end{array}$ & $\begin{array}{c}3.33 \\
(0.38)\end{array}$ & $\begin{array}{l}11.33 \\
(0.38)\end{array}$ & $\begin{array}{l}13.67 \\
(0.32)\end{array}$ & $\begin{array}{l}49.67 \\
(1.34)\end{array}$ \\
\hline $\mathrm{x})$ & $\begin{array}{l}\text { Offals, liver } \\
\text { chicken }\end{array}$ & 0 & 0 & 0 & 0 & 0 & 0 \\
\hline xi) & $\begin{array}{l}\text { Offals, liver } \\
\text { duck }\end{array}$ & 0 & 0 & 0 & $\begin{array}{c}5 \\
(0.16)\end{array}$ & $\begin{array}{c}0.67 \\
\#\end{array}$ & $\begin{array}{c}0.67 \\
\#\end{array}$ \\
\hline xii) & $\begin{array}{l}\text { Offals, liver } \\
\text { geese }\end{array}$ & 0 & 0 & 0 & 0 & 0 & 0 \\
\hline & Total & $\begin{array}{c}16.67 \\
(89.28)\end{array}$ & $\begin{array}{l}94.34 \\
(100)\end{array}$ & $\begin{array}{l}746.67 \\
(86.19)\end{array}$ & $\begin{array}{l}881.67 \\
(29.72)\end{array}$ & $\begin{array}{l}688.99 \\
(16.31)\end{array}$ & $\begin{array}{c}1417.333 \\
(38.38)\end{array}$ \\
\hline 2 & \multicolumn{7}{|l|}{ Live Poultry } \\
\hline i) & Chickens & 0 & 0 & $\begin{array}{l}37.33 \\
(4.31) \\
\end{array}$ & $\begin{array}{c}1025 \\
(34.55)\end{array}$ & $\begin{array}{c}2845.33 \\
(67.38)\end{array}$ & $\begin{array}{c}1907.333 \\
(51.65)\end{array}$ \\
\hline ii) & Ducks & 0 & 0 & $\begin{array}{l}82.33 \\
(9.50)\end{array}$ & $\begin{array}{l}1030.67 \\
(34.75)\end{array}$ & $\begin{array}{l}688.67 \\
(16.31)\end{array}$ & $\begin{array}{c}343.33 \\
(9.29)\end{array}$ \\
\hline iii) & Turkeys & $\begin{array}{c}2 \\
(10.71)\end{array}$ & 0 & 0 & $\begin{array}{l}28.67 \\
(0.97)\end{array}$ & 0 & $\begin{array}{l}24.67 \\
(0.67)\end{array}$ \\
\hline & Total & $\begin{array}{c}2 \\
(10.71)\end{array}$ & 0 & $\begin{array}{c}119.6667 \\
(13.81)\end{array}$ & $\begin{array}{c}2084.33367 \\
(70.27)\end{array}$ & $\begin{array}{c}3534 \\
(83.68)\end{array}$ & $\begin{array}{c}2275.333 \\
(61.61)\end{array}$ \\
\hline & Grand Total & $\begin{array}{l}18.67 \\
(100)\end{array}$ & $\begin{array}{l}94.34 \\
(100)\end{array}$ & $\begin{array}{c}866.34 \\
(100)\end{array}$ & $\begin{array}{l}2966 \\
(100)\end{array}$ & $\begin{array}{l}4223 \\
(100)\end{array}$ & $\begin{array}{c}3692.66 \\
(100)\end{array}$ \\
\hline
\end{tabular}

Figure in parenthesis indicates percentage of total import value of poultry \# indicates negligible share 
Table.4 Net export (Value) of poultry sector from India (1000 US\$)

\begin{tabular}{|l|l|r|r|r|r|r|r|}
\hline & \multicolumn{9}{|c|}{ Net Export (1000 US\$) } \\
\hline S. No. & Items & $\mathbf{1 9 9 6 - 9 8}$ & $\mathbf{1 9 9 9 - 0 1}$ & $\mathbf{2 0 0 2 - 0 4}$ & $\mathbf{2 0 0 5 - 0 7}$ & $\mathbf{2 0 0 8 - 1 0}$ & \multicolumn{2}{|c|}{$\mathbf{1 1 - 1 3}$} \\
\hline $\mathbf{1 .}$ & Eggs, dried & 6959.33 & 3340.67 & 8573 & 6959.33 & 31567.33 & 37451.66 \\
\hline $\mathbf{2 .}$ & Eggs, hen, in shell & 12833.33 & 12413.33 & 26430.33 & 12833.33 & 46270.34 & 40051 \\
\hline $\mathbf{3 .}$ & Eggs, liquid & 4075.99 & 6753 & 13153.67 & 4076 & 840.33 & 2127.34 \\
\hline $\mathbf{4 .}$ & Eggs, other bird, in shell & 0 & 0 & 0 & 0 & 0 & 1618 \\
\hline $\mathbf{5 .}$ & Meat, chicken & 10.33 & 47.67 & 2076 & 10.33 & 1870.33 & 9203.67 \\
\hline $\mathbf{6 .}$ & Meat, chicken, canned & 631.33 & 218.66 & 331.33 & 631.33 & 415.67 & 389.33 \\
\hline $\mathbf{7 .}$ & Meat, duck & 7 & -2.67 & 344.33 & 7 & 581.67 & 691.67 \\
\hline $\mathbf{8 .}$ & Meat, goose and guinea fowl & 0 & 0 & 0 & 0 & 0 & 0.67 \\
\hline $\mathbf{9 .}$ & Meat, turkey & 0 & -1.33 & 323 & 0 & -13.67 & 41.66 \\
\hline $\mathbf{1 0 .}$ & Offals, liver chicken & 0 & 0 & 0 & 0 & 0 & 0 \\
\hline $\mathbf{1 1 .}$ & Offals, liver duck & 0 & 0 & 0 & 0 & -0.67 & -0.67 \\
\hline $\mathbf{1 2 .}$ & Offals, liver geese & 0 & 0 & 0 & 0 & 0 & 0 \\
\hline $\mathbf{1 3 .}$ & Chickens & 328.67 & 312.33 & 412.34 & 328.67 & -2661.63 & -1506.67 \\
\hline $\mathbf{1 4}$ & Ducks & 681 & 769.67 & 590.34 & 681 & -614.00 & -336.67 \\
\hline $\mathbf{1 5 .}$ & Turkeys & -2 & 0 & 1.67 & -2 & 77.67 & 179 \\
\hline $\mathbf{1 6 .}$ & Total & 25524.99 & 23851.33 & 52236 & 25525 & 78333.34 & 89910 \\
\hline
\end{tabular}

Table.5 Growth rate of export and import value of poultry sector

\begin{tabular}{|c|c|c|c|c|c|c|c|}
\hline \multirow{3}{*}{ S. No. } & \multirow{3}{*}{ Items } & \multicolumn{6}{|c|}{ Average Value of Commodity (1000 US\$) } \\
\hline & & \multicolumn{3}{|c|}{ Export } & \multicolumn{3}{|c|}{ Import } \\
\hline & & 1996-2004 & 2005-13 & 1996-2013 & 1996-2004 & 2005-2013 & 1996-2013 \\
\hline 1. & Eggs, dried & -0.05 & 0.08 & 0.10 & $*$ & -0.09 & $-0.29^{f}$ \\
\hline 2. & Eggs, hen, in shell & 0.14 & -0.03 & 0.06 & 1.26 & $\#$ & 0.50 \\
\hline 3. & Eggs, liquid & 0.11 & \# & -0.05 & $0.94^{\mathrm{g}}$ & -0.02 & 0.15 \\
\hline 4. & Eggs, other bird, in shell & - & $*$ & - & - & - & - \\
\hline 5. & Meat, chicken & 1.47 & 0.37 & 0.69 & $*$ & $*$ & $0.37^{\mathrm{m}}$ \\
\hline 6. & Meat, chicken, canned & -0.15 & 0.04 & -0.03 & 0.36 & 0.18 & 0.33 \\
\hline 7. & Meat, duck & 0.82 & $0.01^{\mathrm{b}}$ & 0.35 & 0 & -0.28 & 0 \\
\hline 8. & $\begin{array}{l}\text { Meat, goose and guinea } \\
\text { fowl }\end{array}$ & & $*$ & - & - & - & - \\
\hline 9. & Meat, turkey & $38.79^{\mathrm{a}}$ & -0.22 & $0.01^{\mathrm{d}}$ & $0.15^{\mathrm{h}}$ & -0.21 & $0.08^{\mathrm{n}}$ \\
\hline 10. & Offals, liver chicken & - & - & - & - & - & - \\
\hline 11. & Offals, liver duck & - & - & - & - & $-0.33^{j}$ & $-0.33^{j}$ \\
\hline 12. & Offals, liver geese & - & - & & - & - & - \\
\hline 13. & Chickens & 0.03 & 0.38 & 0.06 & $*$ & $0.07^{\mathrm{b}}$ & $0.40 \mathrm{f}$ \\
\hline 14 & Ducks & -0.09 & $-0.41^{\mathrm{c}}$ & $-0.30^{\mathrm{e}}$ & $0.03^{i}$ & $-0.11^{\mathrm{k}}$ & $0.16^{\circ}$ \\
\hline 15. & Turkeys & $*$ & 1.17 & $0.58^{\mathrm{f}}$ & $*$ & $-0.36^{1}$ & $0^{\mathrm{p}}$ \\
\hline
\end{tabular}

\# indicates negligible growth rate, -indicates no export values, * indicates value available only for single year, a indicates of 2002-03, b indicates from 2006-13, c indicates from 2005-13, d indicates from 2002-13, e indicates from 1996-2012, f indicates from 2003-13, g indicates of 1996-2003, h indicates 1999-2003, i indicates from 200204, $\mathrm{j}$ indicates2006-11, $\mathrm{k}$ indicates 2005-12, $\mathrm{l}$ indicates from 2007-13, $\mathrm{m}$ indicates 2003-07, $\mathrm{n}$ indicates 1999-2013, o indicates 2002-12 p indicates 1998-2013 
Table.6 Variability in export and import of poultry (per cent)

\begin{tabular}{|l|l|r|r|r|r|r|r|}
\hline & & \multicolumn{3}{|c|}{ Export } & \multicolumn{3}{|c|}{ Import } \\
\hline S. No. & Products & $\mathbf{1 9 9 6 - 2 0 0 4}$ & $\mathbf{2 0 0 5 - 1 3}$ & $\mathbf{1 9 9 6 - 2 0 1 3}$ & $\mathbf{1 9 9 6 - 2 0 0 4}$ & $\mathbf{2 0 0 5 - 1 3}$ & $\mathbf{1 9 9 6 - 2 0 1 3}$ \\
\hline & Eggs, dried & 51.13 & 22.20 & 0.76 & 300 & 251.28 & 265.04 \\
\hline $\mathbf{1 .}$ & Eggs, hen, in shell & 54.41 & 22.73 & 0.55 & 167.51 & 43.91 & 89.45 \\
\hline $\mathbf{2 .}$ & Eggs, liquid & 61.45 & 73.28 & 1.03 & 296.77 & 144.0349 & 188.99 \\
\hline $\mathbf{3 .}$ & Eggs, other bird, in shell & - & 300 & 4.24 & - & - & - \\
\hline $\mathbf{4 .}$ & Meat, chicken & 205.54 & 117.45 & 1.60 & 300 & 300 & 337.81 \\
\hline $\mathbf{5 .}$ & Meat, chicken, canned & 56.05 & 28.86 & 0.43 & 134.27 & 54.13 & 104.90 \\
\hline $\mathbf{6 .}$ & Meat, duck & 208.01 & 93.81 & 1.34 & 58.26 & 154.55 & 208.60 \\
\hline $\mathbf{7 .}$ & Meat, goose and guinea fowl & - & 300 & 4.24 & - & - & - \\
\hline $\mathbf{8 .}$ & Meat, turkey & 291.82 & 213.65 & 2.42 & 111.86 & 116.68 & 176.14 \\
\hline $\mathbf{9 .}$ & Offals, liver chicken & - & - & - & - & - & - \\
\hline $\mathbf{1 0}$ & Offals, liver duck & - & - & - & - & 231.38 & 333.72 \\
\hline $\mathbf{1 1}$ & Offals, liver geese & - & - & - & - & - & - \\
\hline $\mathbf{1 2 .}$ & Chickens & 32.56 & 93.29 & 0.63 & 300 & 65.87 & 135.60 \\
\hline $\mathbf{1 3 .}$ & Ducks & 53.53 & 95.96 & 1.04 & 162.42 & 73.71 & 136.22 \\
\hline $\mathbf{1 4}$ & Turkeys & 300 & 145.68 & 2.24 & 300 & 190.87 & 270.27 \\
\hline
\end{tabular}

Table.7 Value of Simpson index

\begin{tabular}{|l|l|l|l|l|l|}
\hline S. No. & \multirow{2}{*}{ Year } & World & India & \\
\cline { 3 - 6 } & & Export & Import & Export & Import \\
\hline 1. & $1996-98$ & 0.66 & 0.68 & 0.64 & 0.44 \\
\hline $\mathbf{2 .}$ & $1999-01$ & 0.66 & 0.68 & 0.63 & 0.47 \\
\hline $\mathbf{3 .}$ & $2002-04$ & 0.69 & 0.70 & 0.65 & 0.48 \\
\hline $\mathbf{4 .}$ & $2005-07$ & 0.68 & 0.69 & 0.52 & 0.70 \\
\hline $\mathbf{5 .}$ & $2008-10$ & 0.68 & 0.67 & 0.53 & 0.51 \\
\hline 6. & $2011-13$ & 0.67 & 0.92 & 0.64 & 0.64 \\
\hline
\end{tabular}

Table.8 Export performance ratio

\begin{tabular}{|c|c|c|c|c|c|c|c|}
\hline & & \multicolumn{6}{|c|}{ Average value of commodities (1000 US\$) } \\
\hline S. No. & Items & $1996-98$ & $1999-01$ & $2002-04$ & $2005-07$ & $2008-10$ & $2011-13$ \\
\hline & Eggs, dried & 29.96 & 20.65 & 19.14 & 43.47 & 44.04 & 49.22 \\
\hline 1. & Eggs, hen, in shell & 5.72 & 6.93 & 6.69 & 7.55 & 6.21 & 5.50 \\
\hline 2. & Eggs, liquid & 10.30 & 19.44 & 14.19 & 0.87 & 0.69 & 1.40 \\
\hline 3. & Eggs, other bird, in shell & 0 & 0 & 0 & 0 & 0 & 2.11 \\
\hline 4. & Meat, chicken & \# & \# & 0.08 & 0.02 & 0.04 & 0.19 \\
\hline 5. & Meat, chicken, canned & 0.23 & 0.07 & 0.04 & 0.03 & 0.04 & 0.03 \\
\hline 6. & Meat, duck & 0.02 & \# & 0.37 & 0.18 & 0.65 & 0.50 \\
\hline 7. & Meat, goose and guinea fowl & 0 & 0 & 0 & 0 & 0 & \# \\
\hline 8. & Meat, turkey & 0 & 0 & 0.06 & 0.11 & 0 & 0.02 \\
\hline 9. & Offals, liver chicken & 0 & 0 & 0 & 0 & 0 & 0 \\
\hline 10. & Offals, liver duck & 0 & 0 & 0 & 0 & 0 & 0 \\
\hline 11. & Offals, liver geese & 0 & 0 & 0 & 0 & 0 & 0 \\
\hline 12. & Chickens & 0.20 & 0.21 & 0.14 & 0.06 & 0.04 & 0.08 \\
\hline 13. & Ducks & 7.87 & 10.40 & 5.81 & 1.03 & 0.51 & 0.03 \\
\hline 14 & Turkeys & 0 & 0 & $\#$ & 0.09 & 0.12 & 0.27 \\
\hline
\end{tabular}




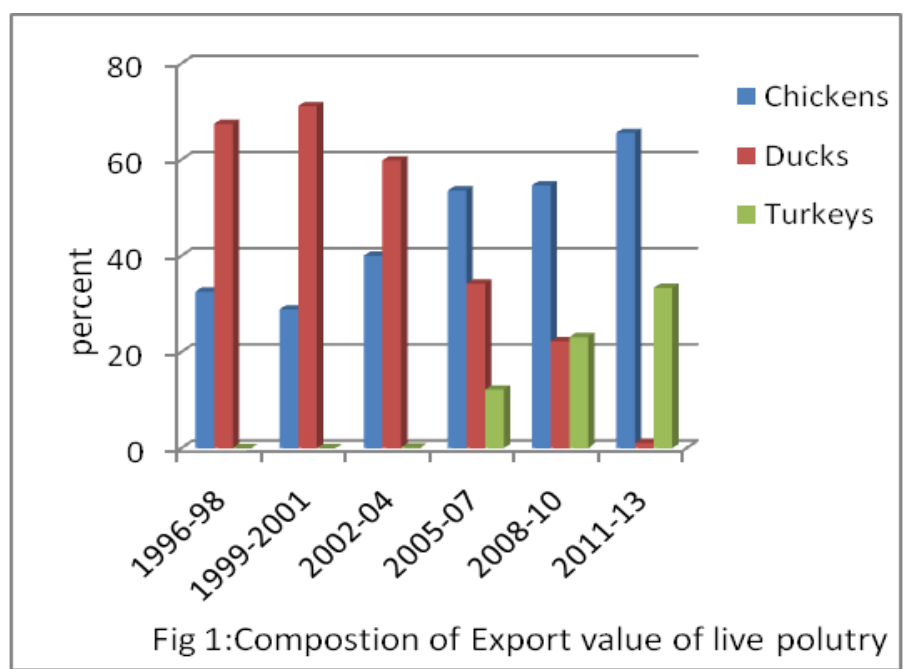

Growth rate of poultry sector

Table 5 reflects the compound annual growth rate of Export and Import value of poultry sector. The highest growth rate in export i.e. 38.79 per cent was registered by meat turkey for period 2002-03. No negative either import or export growth rates were observed for meat chicken and live chicken during study period. However export growth rate of canned chicken meat was found to be negative for 1996-2004. Egg dried, egg hen in shell and egg liquids registered negative growth rate in export for 1996-2004, 2005-13 and 1996-2013 respectively. However egg hen in shell and egg liquid shows positive import growth rate for 1996-2013.

Among live poultry products export value of ducks shows negative growth rate for 19962013 while import growth rates during the same periods for chicken and ducks was found to be positive and no growth was registered in import value of turkey.

\section{Variability in poultry sector trade}

Table 6 shows the coefficient of variation of export and imports of different poultry products in two sub-periods, i.e. from 1996 to 2004 and 2005 to 2013. For period 1996 to 2004 live Turkey export exhibits the maximum variability followed by meat turkey, meat duck and meat

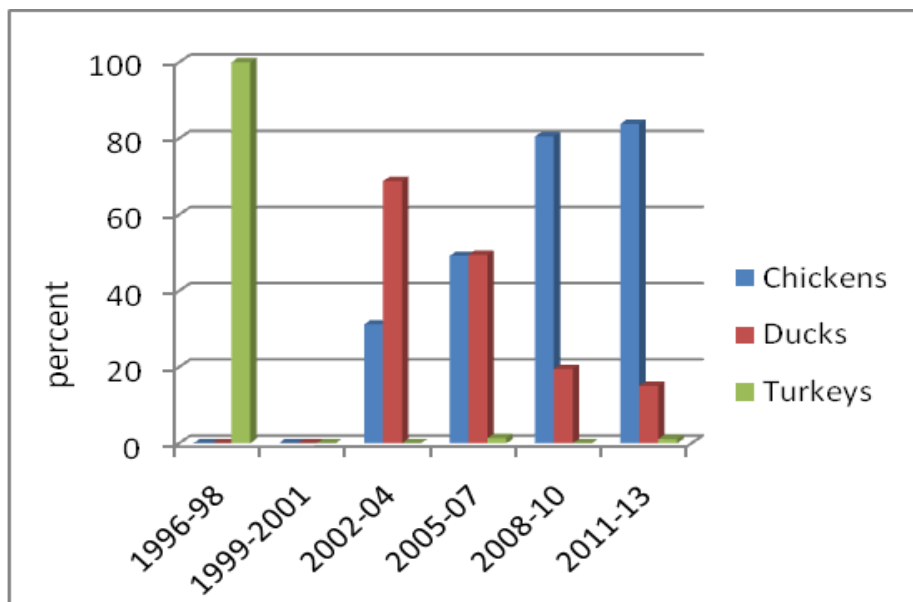

Fig 2: Composition of import value of live poultry

chicken. However the chicken shows the minimum variation. In sub-period 2005-13 Eggs, other birds in shell and meat, goose and guinea fowl shows maximum variation followed by meat turkey, live turkey and meat chicken.

For imports meat chicken exhibits the maximum variability in both sub-period 19962004 and 2005-13. For sub-period 1996-2004 live chicken, live turkey and eggs dried also shows maximum variability followed by egg liquids. For 2005-13 period, after meat chicken egg dried show maximum variability followed by offal, liver duck

\section{Trade diversification}

Export and import diversification for world and India, measured by Simpson index is presented in Table 7. The value of the index near to 1 shows diversification whereas the value of index near to 0 indicates concentration of trade towards some products.

The values of export and import diversification indices for world were relatively higher than that of India showing relatively more trade diversity in world than India. The value to import diversification index for world was 0.92 in T.E. 2013 showing almost complete import diversification of poultry sector. The values of export diversity indices for India were found to 
be moving around 6.3 except for the period 2005-10 in which the value of indices were 0.52 and 0.53. However, the import diversification indices kept fluctuating during the period and varied from 0.44 in T.E.1999 to 0.70 in T.E. 2007 showing most diversification in import of poultry products during T.E. 2007

\section{Comparative advantage of India's poultry product export}

The values export performance ratio (EPR) presented in Table 8 reveals that India has comparative advantage Eggs, dried, Eggs, hen, in shell during the reference period. However India also enjoyed comparative advantage in egg liquids during 1996 to 2004 and for 2011 to 2013 and for duck during 1996 to 2007. The values of EPR in different T.E. for egg dried shows constant decreasing and then increasing trend.

Eggs hen in shell comprises the highest percent of the poultry products exported from India followed by the Egg dried. However, there was no export of Offal during study period. Both Live Turkey and turkey meat comprises almost negligible share in export value of total poultry products from India. No live poultry were imported to India during T.E. 2001.Egg hen in shell comprises highest share in import value of poultry sector while no import of eggs other birds in shell during 1996-2013.Among offals only offals liver ducks, which were imported to India during 2005-13.Share of import and export value of turkey in total value of live poultry import and export of India shows changing status of India from importer of turkey to exporter of turkey. It was found that chicken was the most frequently traded live poultry product. The trend in net export shows that India enjoyed as net exporter of total poultry products. The trend in growth rate shows that egg hen in shell and egg liquid shows positive import growth rate for 1996-2013.Maximum export variability was exhibits by live Turkey for period 1996 to 2004 and maximum imports variability was for meat chicken in both subperiod 1996-2004 and 2005-13. It was found that there is relatively more trade diversity in world than India. The value to import diversification index for world was 0.92 in T.E. 2013 showing almost complete import diversification of poultry sector. Maximum diversification in import of poultry products in India was observed during T.E. 2007. India has comparative advantage Eggs, dried, Eggs, hen, in shell however India lost comparative advantage in egg liquids during 2005 to 2010

\section{References}

APEDA. www.apeda.gov.in

Balassa, B., 1965. Trade Liberalisation and Revealed Comparative Advantage. Manchester School of Economics and Social Studies. 33(2): 99-124.

Bardhan D., 2007. India's trade performance in Livestock and Livestock Products. Indian journal of agricultural economics.62 (3):411-425

FAOSTAT database. www.faostat.fao.org

Kumar Ajani, 2010. Exports of Livestock Products from India: Performance, Competitiveness and Determinants. Agricultural Economics Research Review. 23: $57-67$

Kumar, Anjani, Jabir Ali and Harbir Singh.2001.Trade Performance of Livestock Products in India: Trends, Performance and Competitiveness. Indian Journal of Agricultural Economics. 56, (4):653-667.

\section{How to cite this article:}

Priyanka Agarwal, Rakesh Singh, Harshita Tewari and Rekha Rani. 2017. India's Trade Performance in Poultry Products. Int.J.Curr.Microbiol.App.Sci. 6(10): 451-460. doi: https://doi.org/10.20546/ijcmas.2017.610.055 